\title{
Infarto Agudo do Miocárdio Associado a Ponte Miocárdica
}

\author{
Vinicius Esteves ${ }^{1}$, Roberto Ramos Barbosa ${ }^{1}$, J. Ribamar Costa Jr. ${ }^{1}$, Rodolfo Staico ${ }^{1}$, \\ Fausto Feres ${ }^{1}$, Amanda G. M. R. Sousa', J. Eduardo Sousa ${ }^{1}$
}

\section{RESUMO}

Paciente do sexo masculino, 73 anos, hipertenso, dislipidêmico e ex-tabagista, internado com quadro de infarto agudo do miocárdio sem supradesnivelamento do segmento ST. Cineangiocoronariografia demonstrou ponte miocárdica em artéria descendente anterior, sem lesões obstrutivas em outros territórios da circulação coronária. Em decorrência da elevação de marcadores de necrose miocárdica e do surgimento de supradesnivelamento transitório do segmento ST em parede anterior, na vigência de tratamento clínico otimizado, realizou-se ultrassom intracoronário, que evidenciou lesão ulcerada no território da ponte miocárdica, seguido de angioplastia com implante de stent, com sucesso. A evolução foi satisfatória após o procedimento e o paciente recebeu alta hospitalar.

DESCRITORES: Ponte miocárdica. Ultrassom. Angioplastia transluminal percutânea coronária. Stents. Isquemia.

\begin{abstract}
Acute Myocardial Infarction Associated to Myocardial Bridging

We report the case of a 73-year-old man, with a history of hypertension, dyslipidemia, and previous smoking, admitted with a non-ST segment elevation myocardial infarction. Coronary angiography showed a myocardial bridge in the left anterior descending artery, with no obstructive lesions in other coronary arteries. Because of the raise in serum markers of myocardial necrosis and the development of transient ST segment elevation in the anterior wall, despite optimized medical treatment, we performed intravascular ultrasound, which showed an ulcerated lesion in the myocardial bridge territory, which was followed by a successful coronary angioplasty with stent implantation. The patient had a favorable course and was discharged from the hospital.
\end{abstract}

KEY-WORDS: Myocardial bridging. Ultrasonics. Angioplasty, transluminal, percutaneous coronary. Stents. Ischemia.

Relatamos um caso de ponte miocárdica com apresentação clínica adversa e refratariedade ao tratamento clínico, que evoluiu satisfatoriamente após angioplastia coronária com implante de stent.

\section{RELATO DO CASO}

Paciente do sexo masculino, 73 anos, com antecedentes de hipertensão arterial, dislipidemia e tabagismo, admitido no pronto-socorro de nossa instituição com história de precordialgia em opressão iniciada em repouso com duração de uma hora, de forte intensidade, acompanhada de sudorese, sem irradiação, contínua e sem alívio com analgésico comum.

Ao exame físico encontrava-se com pressão arterial de 142/81 mmHg e frequência cardíaca de 86 bpm. Não havia alterações significativas no exame segmentar.

O eletrocardiograma de 12 derivações apresentava ritmo sinusal com alteração de repolarização (onda $T$ bifásica) de V1 a V4 (Figura 1). A creatina quinase fração $M B(C K-M B)$ atividade e a troponina elevaram-se

\footnotetext{
1 Instituto Dante Pazzanese de Cardiologia - São Paulo, SP, Brasil. Correspondência: Vinicius Esteves. Av. Dr. Dante Pazzanese, $500-$ Vila Mariana - São Paulo, SP, Brasil - CEP 04012-180

E-mail: vinasp@hotmail.com

Recebido em: 26/9/2010 • Aceito em: 28/11/2010
} 


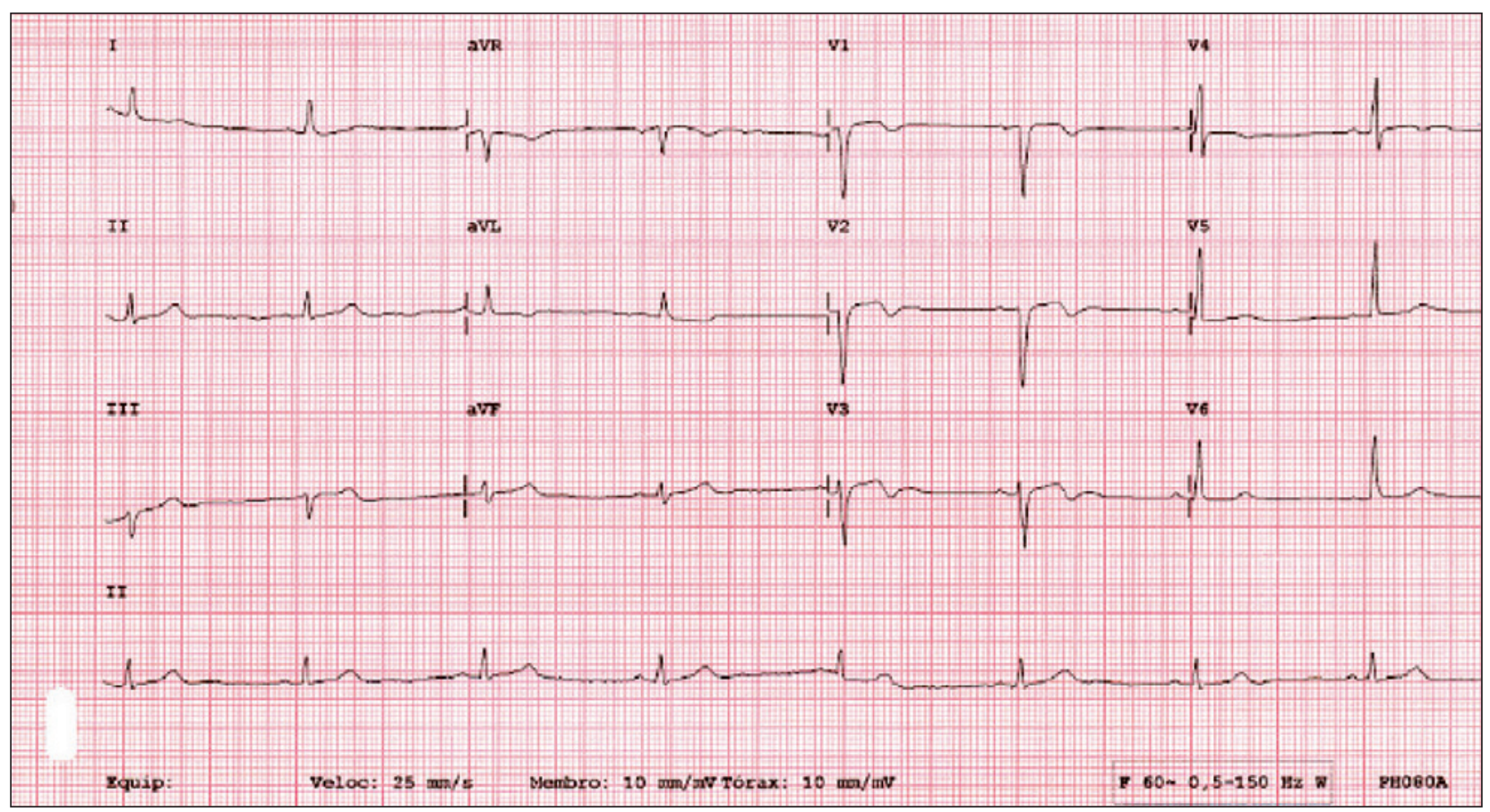

Figura 1 - Eletrocardiograma inicial demonstrando alteração de repolarização ventricular em derivações V1 a V4.

a partir da segunda dosagem. Como terapia farmacológica, foram introduzidos enoxaparina $1 \mathrm{mg} / \mathrm{kg}$ a cada 12 horas, ácido acetilsalicílico $300 \mathrm{mg}$, clopidogrel $300 \mathrm{mg}$, captopril $25 \mathrm{mg}$ a cada 8 horas e atenolol $50 \mathrm{mg}$, com estabilização clínica e controle da dor.

O paciente apresentou recorrência da dor precordial nas primeiras 48 horas de evolução, que piorava com a infusão de nitroglicerina endovenosa. Observouse pico de CK-MB atividade no segundo dia de evolução $(11 \mathrm{U} / \mathrm{l}$; normal $<6 \mathrm{U} / \mathrm{l})$ e pico de troponina no terceiro dia de evolução (7 ng/ml; normal < 0,5 ng/ml).

Após 48 horas da admissão hospitalar foi realizada cineangiocoronariografia, que evidenciou ponte miocárdica em terço médio da artéria descendente anterior (Figura 2) e ausência de lesões obstrutivas em outras artérias coronárias. A ventriculografia esquerda demonstrou função contrátil preservada.

O paciente foi mantido em tratamento clínico na Unidade Coronária, com pressão arterial controlada e frequência cardíaca mantida entre 50 bpm e 60 bpm, à custa de betabloqueador, sendo suspensa a nitroglicerina endovenosa. Mesmo assim, houve novos episódios de precordialgia, com a detecção de supradesnivelamento transitório do segmento ST de V1 a V3 no quarto dia de evolução (Figura 3).

Posteriormente, como método complementar de avaliação, foi realizada angiotomografia de coronárias, demonstrando feixes musculares envolvendo o terço médio da artéria descendente anterior, com profundidade discreta, e imagem sugestiva de lesão estenosante também nesse segmento da artéria descendente anterior (Figura 4).

Ultrassom intracoronário foi realizado no oitavo dia de internação, que evidenciou placa ulcerada com excentricidade, lago lipídico e remodelamento positivo no segmento da ponte miocárdica da artéria descendente anterior (Figura 5). Em decorrência da refratariedade dos sintomas, mesmo com tratamento clínico otimizado, optou-se pela intervenção coronária percutânea. O paciente foi submetido a angioplastia coronária por via radial com implante de stent Liberté $^{\mathrm{TM}}$ 3,5 x $20 \mathrm{~mm}$ (Boston Scientific, Natick, Estados Unidos), com sucesso angiográfico, sendo a aposição das hastes e a expansão adequada do stent confirmadas por ultrassom intracoronário pós-procedimento.

O paciente apresentou evolução satisfatória após a intervenção, recebendo alta no dia seguinte em uso de aspirina $100 \mathrm{mg}$, clopidogrel $75 \mathrm{mg}$, atenolol $50 \mathrm{mg}$, enalapril $20 \mathrm{mg}$ a cada 12 horas e sinvastatina $40 \mathrm{mg}$.

\section{DISCUSSÃO}

O comportamento clínico e o tratamento das pontes miocárdicas ainda requerem elucidações. Há, inclusive, grande discrepância a respeito de sua incidência, com valores díspares de acordo com o método diagnóstico empregado, angiográfico vs. patológico. ${ }^{2}$ Muito se avançou em relação à avaliação dos mecanismos de isquemia por meio do ultrassom e reserva de fluxo coronários, embora ainda não exista consenso. ${ }^{7}$ 

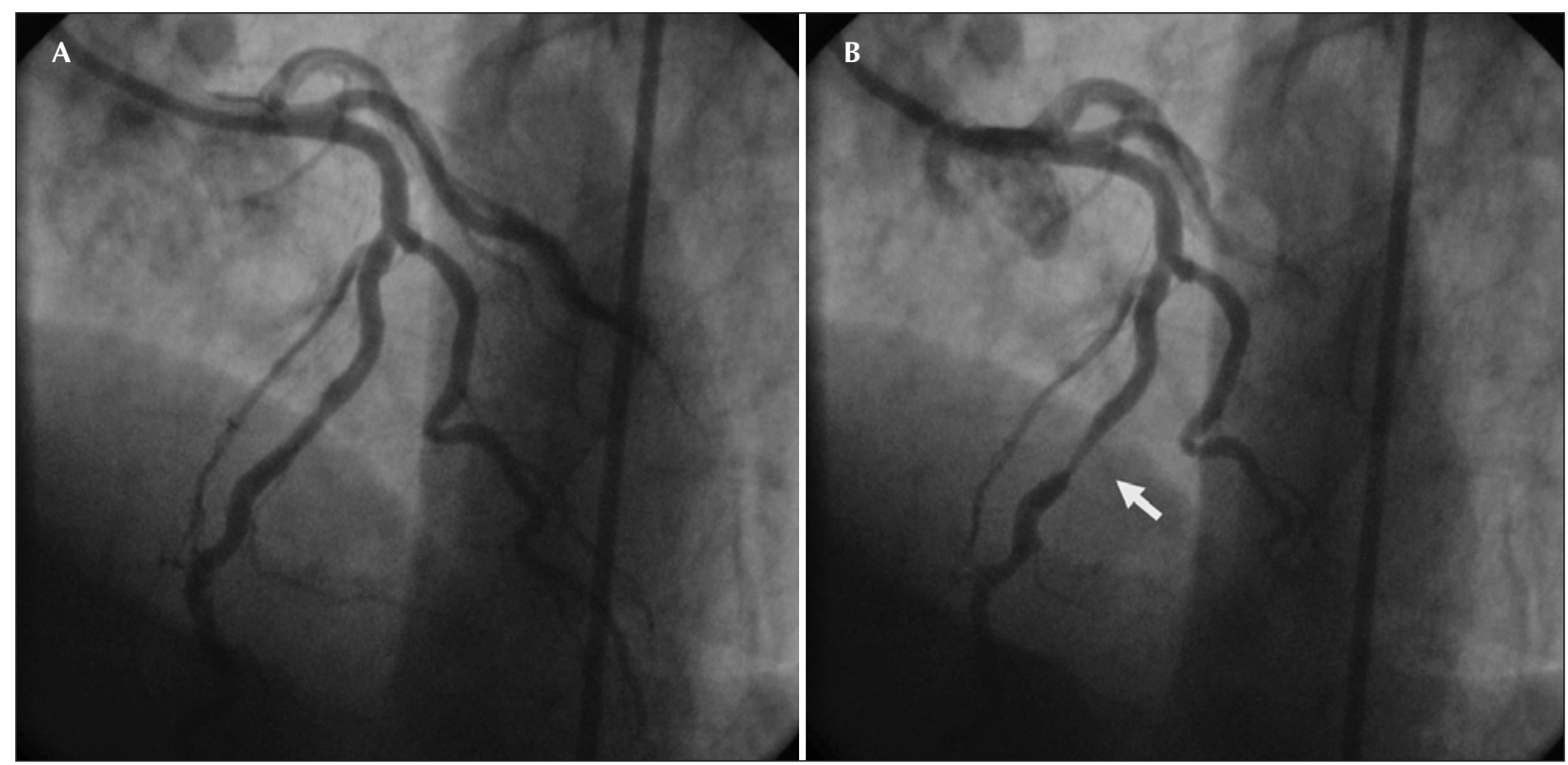

Figura 2 - Cineangiocoronariografia demonstrando, em projeção oblíqua anterior esquerda, respectivamente em diástole (A) e em sístole (B), ponte miocárdica em terço médio da artéria descendente anterior (seta) e ausência de lesões obstrutivas em outras artérias coronárias.

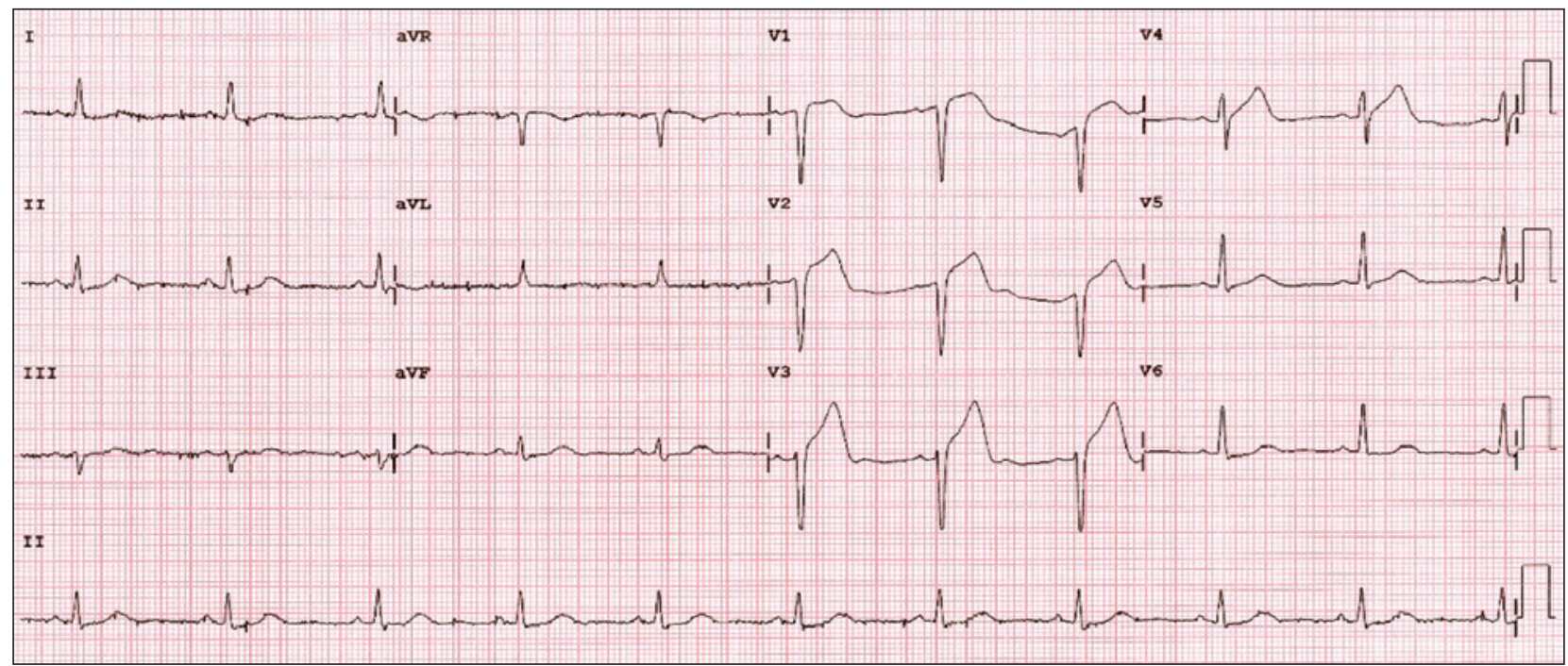

Figura 3 - Eletrocardiograma evolutivo demonstrando supradesnivelamento transitório do segmento ST de V1 a V3.

Ainda que qualquer artéria coronária epicárdica principal possa ser afetada, o envolvimento da artéria descendente anterior é o mais comum. Estudos angiográficos demonstram prevalência de ponte miocárdica de $1,7 \%$ (variando de $0,5 \%$ a $12 \%$ ), acometendo quase sempre a artéria descendente anterior. ${ }^{2,4,8-10}$

A injeção intracoronária de nitroglicerina tende a aumentar a intensidade da compressão, provavelmente em decorrência do reflexo simpático de estímulo contrátil e/ou da menor pressão intraluminal na artéria coronária. ${ }^{11}$ Com isso, o uso rotineiro de nitrato nas coronariografias diagnósticas realizadas por via transradial pode elevar consideravelmente a incidência de ponte miocárdica observada angiograficamente. ${ }^{2,12}$ Porém, na maioria dos casos, não se detecta repercussão clínica do achado angiográfico de ponte miocárdica.

Os estudos existentes, todos retrospectivos e com pequeno número de pacientes, apontam para uma evolução benigna dos portadores de ponte miocárdica. Entretanto, a documentação de angina, espasmo coronário, arritmias ventriculares, infarto agudo do miocárdio e morte súbita em pacientes com ponte miocárdica 


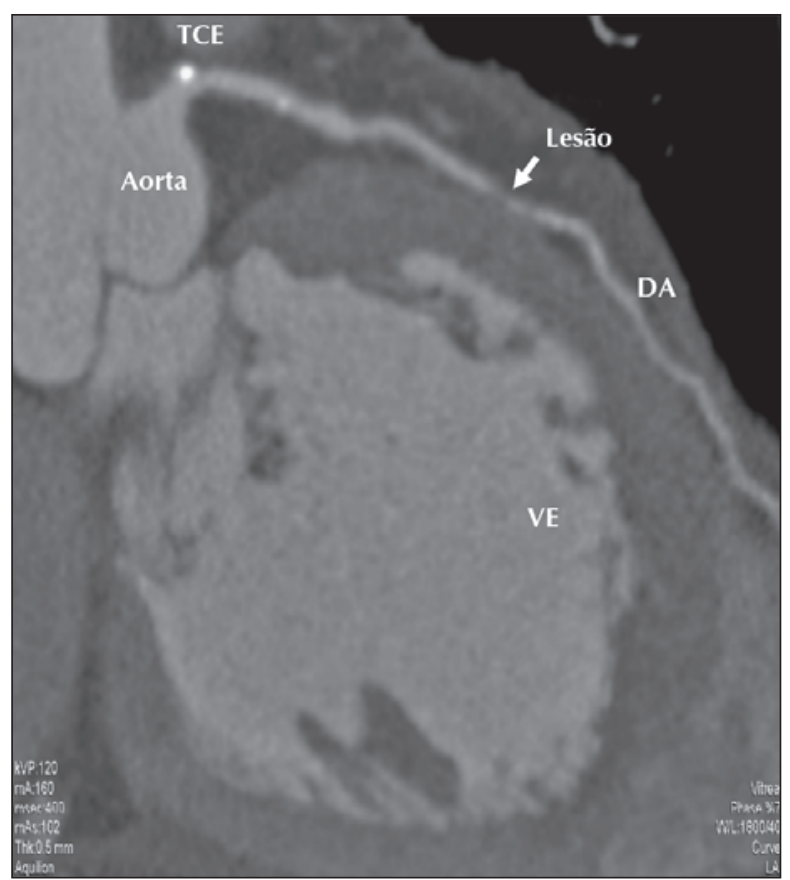

Figura 4 - Angiotomografia coronária demonstrando feixes musculares envolvendo o terço médio da artéria descendente anterior, com profundidade discreta, e imagem sugestiva de lesão estenosante nesse segmento da artéria descendente anterior (seta). DA = artéria descendente anterior; $\mathrm{TCE}=$ tronco da coronária esquerda; $\mathrm{VE}=$ ventrículo esquerdo.

indica a provável existência de um subgrupo de pacientes com maior risco de eventos. ${ }^{6}$ A ausência de estudos prospectivos, controlados, dificulta o estabelecimento definitivo dos fatores prognósticos dessa condição. Em geral, pacientes sintomáticos recebem tratamento clínico com betabloqueadores e/ou antagonistas dos canais de cálcio. ${ }^{2}$ Intervenção percutânea com uso de stents ${ }^{13}$ ou tratamento cirúrgico (miotomia ou cirurgia de revascularização miocárdica) ${ }^{14}$ são destinados apenas à minoria de pacientes que persistem com sintomas ou com provas funcionais positivas apesar do tratamento clínico otimizado.

No caso apresentado, o uso de ultrassonografia intracoronária associada a cineangiocoronariografia demonstrou existência de lesão coronária com sinais de instabilidade (placa ulcerada) no segmento envolvido pela ponte miocárdica. Esse fato, associado à instabilidade clínica, a despeito do tratamento clínico adequado, reforçou a indicação de intervenção coronária percutânea.

Clinicamente, pontes miocárdicas podem estar presentes em indivíduos com ou sem doença coronária, incluindo lesões no mesmo vaso, sem relação com a ponte. Entretanto, há suspeita de que a presença de ponte miocárdica possa favorecer o avanço da aterosclerose coronária. ${ }^{15}$ Alguns estudos demonstraram espessamento intimal concêntrico sob as pontes

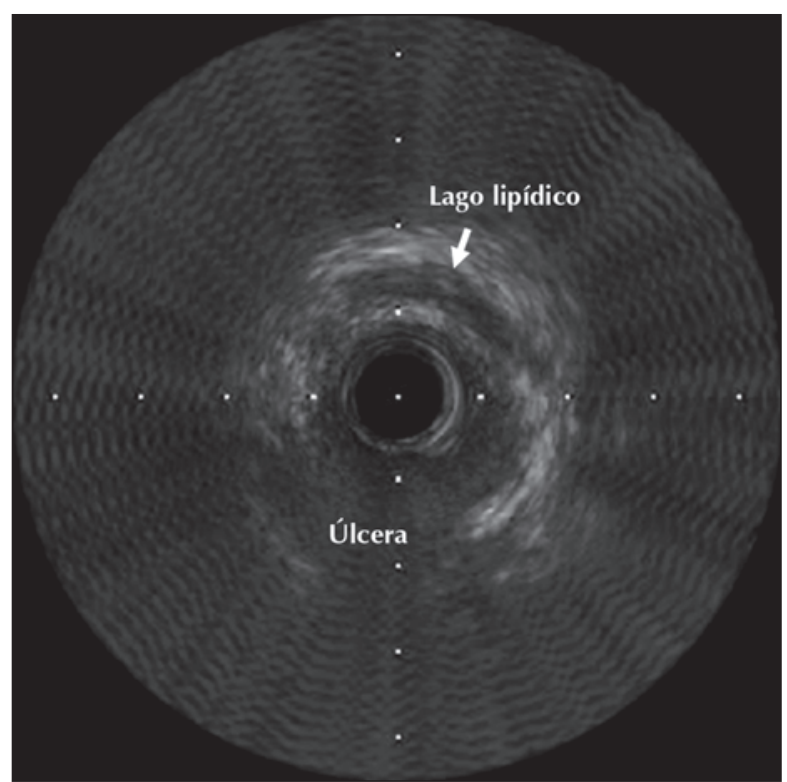

Figura 5 - Ultrassom intracoronário evidenciando placa ulcerada, excêntrica, com lago lipídico e remodelamento positivo, no segmento da ponte miocárdica da artéria descendente anterior.

miocárdicas e aumento de placas ateroscleróticas proximais às pontes. Isso foi observado tanto com o estudo anatomopatológico como com o uso do ultrassom intracoronário. ${ }^{8,16}$

Estudos utilizando ultrassom intracoronário em pontes miocárdicas observaram, além da compressão sistólica característica com retardo do relaxamento na fase diastólica, aumento da velocidade média de fluxo após administração intracoronária de papaverina, um potente vasodilatador coronário. A variação da área luminal é de $40 \pm 25 \%$ nos segmentos de ponte miocárdica vs. $9 \pm 7 \%$ nos segmentos normais. Nenhuma lesão aterosclerótica foi detectada pelo ultrassom intracoronário dentro ou distal aos segmentos de ponte miocárdica. Contudo, lesões ateroscleróticas foram observadas em segmentos proximais às pontes miocárdicas em 12 de 14 pacientes. ${ }^{16}$ A reserva de fluxo coronário distal à ponte varia de 2 a 2,6 (normal > 3), possivelmente em decorrência de aterosclerose proximal ou retardo da dilatação coronária na diástole. ${ }^{7}$ Esses achados podem explicar a isquemia demonstrada em alguns desses pacientes. .,17 $^{2}$

No panorama atual, o ultrassom intracoronário parece ser um instrumento útil e acessível para a identificação de fatores prognósticos em pacientes selecionados portadores de pontes miocárdicas, necessitando, entretanto, validação em populações maiores.

\section{CONFLITO DE INTERESSES}

Os autores declararam inexistência de conflito de interesses relacionado a este manuscrito. 


\section{REFERÊNCIAS}

1. Reyman HC. Disertatis de vasis cordis propiis. Bibl Anat. 1737;2:366.

2. Mohlenkamp S, Hort W, Ge J, Erbel R. Update on myocardial bridging. Circulation. 2002;106(20):2616-22.

3. Noble J, Bourassa MG, Petitclerc R, Dyrda I. Myocardial bridging and milking effect of the left anterior descending coronary artery: normal variant or obstruction? Am J Cardiol. 1976;37(7):993-9.

4. Faruqui AM, Maloy WC, Felner JM, Schlant RC, Logan WD, Symbas P. Symptomatic myocardial bridging of coronary artery. Am J Cardiol. 1978;41(7):1305-10.

5. Morales AR, Ramanelli R, Boucek R. The mural left descending coronary artery, strenuous exercise and sudden death. Circulation. 1980;62(2):230-7.

6. Hayashi T, Ishikawa K. Myocardial bridge: harmless or harmful. Intern Med. 2004;43(12):1097-8.

7. Bourassa MG, Butnaru A, Lesperance J, Tardif JC. Symptomatic myocardial bridges: overview of ischemic mechanisms and current diagnostic and treatment strategies. J Am Coll Cardiol. 2003;41(3):351-9.

8. Kramer JR, Kitazume H, Proudfit WL, Sones FM Jr. Clinical significance of isolated coronary bridges: benign and frequent condition involving the left anterior descending artery. Am Heart J. 1982;103(2):283-8.

9. Channer KS, Bukis E, Hartnell G, Rees JR. Myocardial bridging of the coronary arteries. Clin Radiol. 1989;40(4):355-9.
10. Irvin RG. The angiographic prevalence of myocardial bridging in man. Chest. 1982;81(2):198-202.

11. Hongo $\mathrm{Y}$, Tada $\mathrm{H}$, Ito $\mathrm{K}$, Yasumura $\mathrm{Y}$, Miyatake $\mathrm{K}$, Yamagishi $M$. Augmentation of vessel squeezing at coronary-myocardial bridge by nitroglycerin: study by quantitative coronary angiography and intravascular ultrasound. Am Heart J. 1999; 138(2 Pt 1):345-50

12. Santos LM, Araújo EC, Sousa LNL. Ponte miocárdica multiarterial: apresentações clínica e anatômica incomuns. Arq Bras Cardiol. 2007;88(4):e73-5.

13. Klues HG, Schwartz ER, vom Dahl J, Reffelman R, Minartz J, Potthast K, et al. Disturbed intracoronary hemodynamics in myocardial bridging: early normalization by intracoronary stent placement. Circulation. 1997;96(9):2905-13.

14. Hill RC, Chitwood WR Jr, Bashore TM, Sink JD, Cox JL, Wechsler AS. Coronary flow and regional function before and after supraarterial myotomy for myocardial bridging. Ann Thorac Surg. 1981;31(2):176-81.

15. Bennett JM, Blomerus P. Thallium-201 scintigraphy perfusion defect with dipyridamole in a patient with a myocardial bridge. Clin Cardiol. 1988;11(4):268-70.

16. Ge J, Erbel R, Rupprecht HJ, Koch L, Kearney P, Görge G, et al. Comparison of intravascular ultrasound and angiography in the assessment of myocardial bridging. Circulation. 1994; 89(4):1725-32.

17. Angelini P, Velasco JA, Flamm S. Coronary anomalies: incidence, pathophysiology, and clinical relevance. Circulation. 2002; 105(20):2449-54 . 